\title{
Automatic Identification of Atrial Fibrillation by Spectral Analysis of Fibrillatory Waves
}

\author{
Agnese Sbrollini, Krizia Cicchetti, Alessia De Martinis, \\ Ilaria Marcantoni, Micaela Morettini, Laura Burattini \\ Università Politecnica delle Marche, Ancona, Italy
}

\begin{abstract}
A heart affected by atrial fibrillation $(A F)$ presents atrial cells that depolarize in many sites, generating a chaotic electrical activity. On the electrocardiogram (ECG), this activity reflects in the appearance of fibrillatory $(F)$ waves, consisting of low-amplitude oscillations at 4-10 Hz. Aim of the present study is to propose an automatic AF identification method based on $F$-wave frequency analysis in $10 \mathrm{~s}$ ECGs. To this aim, 10 $s$ ECG from 90 healthy subjects $(\mathrm{HSs})$ and $50 \mathrm{AF}$ patients (AFPs) were considered. ECGs were processed by the segmented beat modulation method to reduce components in the F-wave band. Then, the power spectral density (PSD) was computed and the $F$-wave frequency ratio $(F W F R)$, defined as the ratio between the spectral area in the $F$-wave frequency band and the total spectral area, was computed. FWFR ability to discriminate AFPs from $H S s$ was evaluated by analyzing the area under the curve $(A U C)$ of the receiver operating characteristic, and by computation of sensitivity, specificity and accuracy. FWFR values were higher in AFPs than in HSs $\left(P<10^{-11}\right)$. AUC was at least $85 \%$, whereas sensitivity, specificity and accuracy were at least $84 \%, 69 \%$ and $81 \%$, respectively. In conclusion, $F$-wave frequency evaluation by FWFR represents a promising clinical tool to automatically identify $A F$.
\end{abstract}

\section{Introduction}

Atrial Fibrillation (AF) is a supraventricular arrhythmia that is expected to afflict millions of people worldwide by 2060 [1]. In normal sinus rhythm, a cardiac contraction regularly occurs thanks to an electrical impulse that auto-generates in the sinus atrial node, the native pacemaker, and then propagates in the heart through a specific pathway. From the sinus atrial node, the impulse propagates in atrial cells before reaching the atrioventricular node, where it reorganizes before spreading through ventricular cells. When stimulated by an electrical impulse, each cardiac cell initially depolarizes, generating its contraction, and then repolarizes, generating its relaxation. Thus, the combined electrical activity of all cells in the heart generates its organized contraction and relaxation. The electrocardiographic signal (ECG) is a graphical representation of the electrical activity of all cardiac cells. Normally, it's a pseudo periodic signal characterized by the sequence of typical waveforms that are the $\mathrm{P}$ wave, which reflects atria depolarization; the QRS complex, which reflects ventricles depolarization and hides atria repolarization; and the $\mathrm{T}$ wave, which reflects ventricles repolarization.

In a heart affected by AF, the sinus atrial node is no longer able to trigger atrial depolarization [2] so that atrial cells start to depolarize in many sites, generating a chaotic electrical activity. As a consequence, heart rhythm during $\mathrm{AF}$ is, on average, faster than sinus rhythm, and much more irregular [2]. In the ECG, AF is reflected by the Pwave disappearance and by appearance of the fibrillatory (F) waves, which are low-amplitude and high-frequency oscillations around baseline.

Visual [3] as well as automatic algorithms [4-7] for AF identification from ECG typically rely on the P-wave absence and the irregularity of the rhythm. In computerized applications, however, this approach, has several limitations. P-wave absence is a condition reflected also in other arrhythmias (e.g. PVC). P-wave segmentation is still challenging due to $\mathrm{P}$-wave low amplitude. Automatic algorithms are usually implemented with the aim of finding the P-wave and do not confirm the P-wave absence [8]. Eventually, automatic quantification of the rhythm irregularity needs long-term ECGs, so that algorithms based on this criterion cannot be applied to standard $10 \mathrm{~s}$ ECG.

Aim of the present study is to propose an automatic AF identification method based on F-wave frequency analysis in $10 \mathrm{~s}$ ECGs. Indeed, F-wave frequency content is mostly included in the $4-10 \mathrm{~Hz}$ band [9], which does not contain ECG fundamental frequency (typically falling in the 1-2 Hz band). Previous studies have investigated Fwave frequency content $[9,10]$, but not to provide an automatic tool to support AF diagnoses. 


\section{Data and Methods}

\subsection{Clinical Data}

Clinical data consisted of 10 s ECGs from 90 healthy volunteer subjects (HSs) and $50 \mathrm{AF}$ patients (AFPs).

HS ECGs were extracted from the "The ECG-ID Database" by Physionet [11,12]. Specifically, they represent the first $10 \mathrm{~s}$ of the first acquired ECG, after filtration for noise removal of each subject $[11,12]$. Signal sampling frequency is $500 \mathrm{~Hz}$.

AFP ECGs were extracted from "The AF Termination Challenge Database" by Physionet [12,13]. Specifically, they represent the first $10 \mathrm{~s}$ of 20 recordings from the learning set and of 30 recordings from the test set $\mathrm{A}$, selected in order to have only one ECG for each patient. All signals were originally sampled at $128 \mathrm{~Hz}$ and annotated for R-peak localization.

\subsection{Data Processing and Feature Extraction}

HS ECGs were resampled at $128 \mathrm{~Hz}$ (to match AFP ECGs sampling frequency) and submitted to the PanTompkins' algorithm [14] for R-peak detection. RRinterval sequences of both groups were used to compute heart rate (HR; bpm), computed as 60/mean RR interval, and heart-rate variability (HRV; $\mathrm{ms}$ ), computed as RRinterval standard deviation.

ECGs of both groups were preprocessed for noise (bidirectional $3^{\text {rd }}$-order Butterworth filter; cut-off frequencies: $0.5-45 \mathrm{~Hz}$ ) before being submitted to the segmented-beat modulation method (SBMM) $[15,16]$. SBMM is a template-based filtering technique that permits to extract the fundamental ECG waveform (FECG) from an ECG possibly affected by baseline wanderings, respiration modulation, ECG-morphology variability and $\mathrm{F}$ waves, the summation of which represents the residual ECG waveform (RECG). Thus, RECG can be obtained as follow:

$$
\mathrm{RECG}=\mathrm{ECG}-\mathrm{FECG} .
$$

Power spectral density (PSD) of RECG was computed according to the following four methods:

- PSD estimation via periodogram method (FFT). It is computed by using the Fast Fourier Transform, with a rectangular window (13.3 $\mathrm{dB}$ attenuation).

- PSD estimation via Welch's method (WLC). RECG was divided into 8 segments with $50 \%$ overlap. The periodogram was computed for each segment using a Hamming window (42.5 dB attenuation) and all the resulting periodograms are averaged to compute the final PSD estimation.

- PSD estimation via Yule-Walker's method (YWK). The PSD was estimated using a $25^{\text {th }}$-order (experimentally determined) autoregressive model.

- PSD estimation via the Thomson multitaper method (THM). The PSDs of mutually orthogonal windows (tapers) were averaged and opportunely weighted with their eigenvalues. The tapers are Slepian sequences (time-half bandwidth equal to 4).

Presence of F-wave component in each spectrum was quantified by computation of the F-wave frequency ratio (FWFR), a new spectral index defined as the ratio between the spectral area in the F-wave frequency band $(4-10 \mathrm{~Hz})$ and the total spectral area.

\subsection{Statistics}

HR, HRV and FWFR distributions were described in terms of $50^{\text {th }}\left[25^{\text {th. }} ; 75^{\text {th }}\right]$ percentiles and compared using the Wilcoxon Rank-Sum test for equal medians. Statistical significance was set at 0.05 .

The receiver operating characteristic (ROC) was analyzed in order to assess FWFR clinical utility in discriminating AFPs from HSs. Area under the curve (AUC) and 95\% confidence interval (CI) of different ROCs were evaluated and compared using the DeLong's test. The classification error $(\varepsilon)$ was defined as:

$$
\varepsilon=2 \cdot \mathrm{FN}+\mathrm{FP}
$$

where FP and FN are false-positive and false-negative classifications, respectively. Sensitivity (SE), specificity (SP) and accuracy (ACC) were computed based on the FWFR optimal threshold (OTHR), determined in correspondence of the ROC point that minimized $\varepsilon$.

\section{Results}

All considered features were significantly higher in AFPs than in HSs (Table 1). FWFR-based features, however, differed more significantly (lower $P$ value) than HR and HRV.

Table 1: Features comparison between groups $\left(50^{\text {th }}\left[25^{\text {th }} ; 75^{\text {th }}\right]\right.$ percentiles $)$.

\begin{tabular}{lcccc}
\hline & & HSs & AFPs & P-value \\
\hline HR (bpm) & & 76 & 86 & $<10^{-3}$ \\
& & {$[69 ; 85]$} & {$[72 ; 96]$} & \\
\hline HRV (ms) & & 48 & 121 & $<10^{-11}$ \\
& & {$[29 ; 77]$} & {$[95 ; 196]$} & \\
& FFT & 19 & 34 & $<10^{-11}$ \\
& & {$[14 ; 25]$} & {$[28 ; 42]$} & \\
\cline { 2 - 5 } FWFR (\%) & WLC & 19 & 35 & $<10^{-11}$ \\
& & {$[13 ; 27]$} & {$[29 ; 42]$} & \\
& YWK & 20 & 36 & $<10^{-11}$ \\
& & {$[15 ; 26]$} & {$[31 ; 44]$} & \\
& THM & 18 & 35 & $<10^{-11}$ \\
& & {$[13 ; 25]$} & {$[28 ; 42]$} & \\
\hline
\end{tabular}




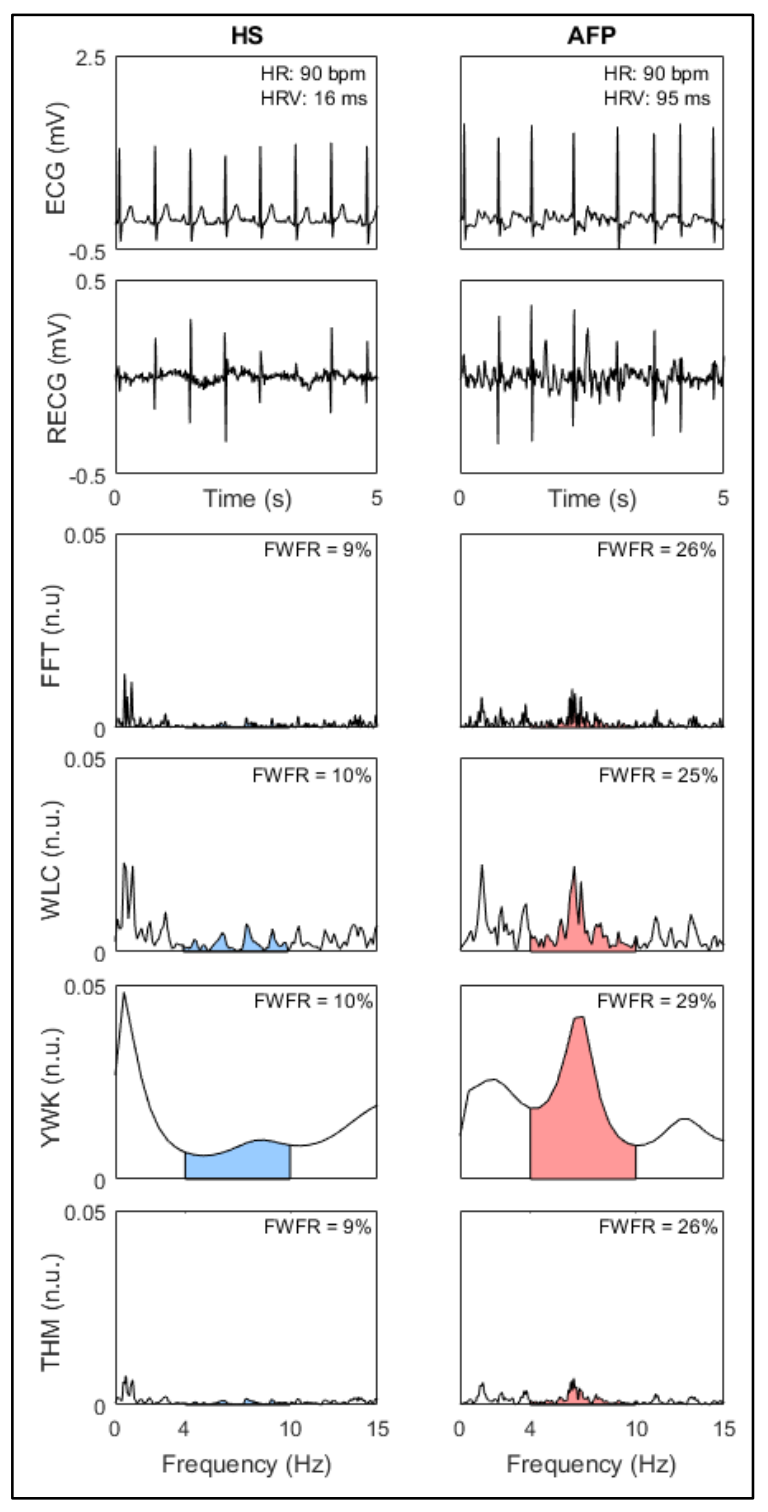

Figure 1. Examples of data processing in a HS (left column of panels) and an AFP (right column of panels). PSD area in F-wave frequency band $(4-10 \mathrm{~Hz})$ is higher in the AFP (red area) than in the HS (blue area).

Table 2. ROC analysis

\begin{tabular}{ccccc}
\hline & FFT & WLC & YWK & THM \\
\hline AUC (\%) & 86 & 85 & 86 & 86 \\
\hline CI (\%) & $80-93$ & $78-93$ & $79-93$ & $79-93$ \\
\hline OTHR (\%) & 25 & 23 & 27 & 25 \\
\hline SE (\%) & 84 & 90 & 84 & 86 \\
\hline SP (\%) & 78 & 69 & 77 & 74 \\
\hline ACC (\%) & 82 & 82 & 81 & 82 \\
\hline
\end{tabular}

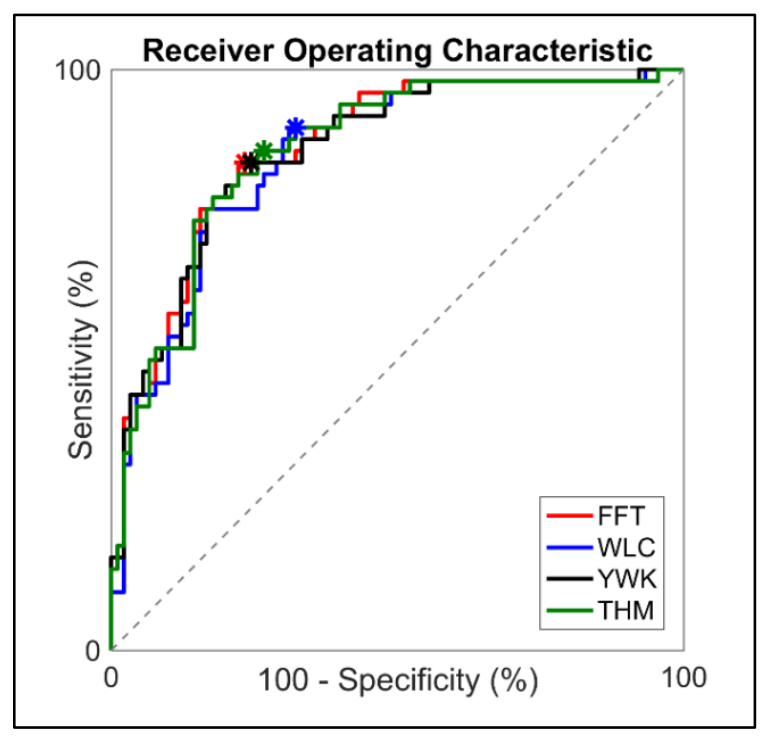

Figure 2. ROCs for each PSD estimation method. Stars identify points in which the OTHRs were determined.

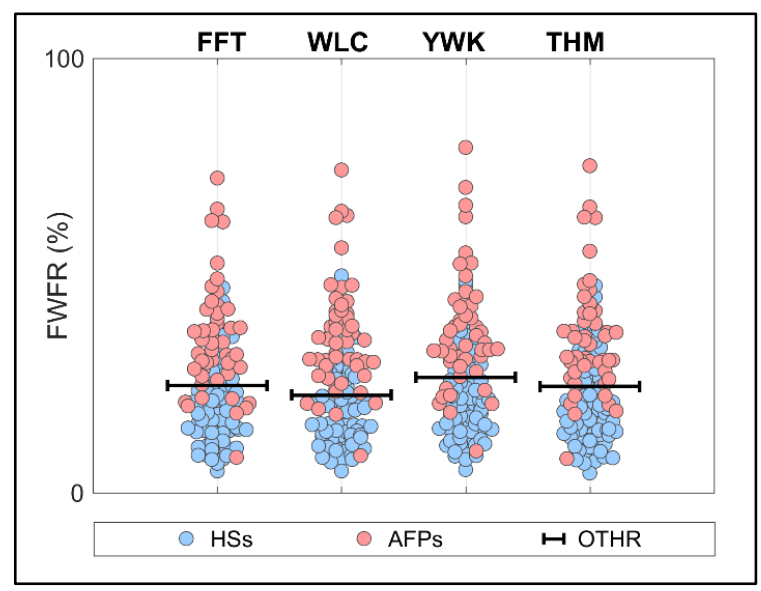

Figure 3. FWFR distributions for each PSD estimation method.

Figure 1 depicts representative examples of data processing in a HS and an AFP. In RECG of the AF, presence of F-waves is clearly visible and reflected in spectral peaks in the $4-10 \mathrm{~Hz}$ band. Results relative to ROC analysis are reported in Table 2 and depicted in Figure 2. All AUC values were at least $85 \%$, and not statistically different from one another. Figure 2 and Figure 3 show OTHR determination. Accordingly, SE, SP and ACC were at least $84 \%, 69 \%$ and $81 \%$, respectively.

\section{Discussion}

The present study proposed automatic AF identification based on F-wave frequency analysis in $10 \mathrm{~s}$ ECGs. At first the ECG was processed using the SBMM 
$[15,16]$ in order to reduce as much as possible ECG frequency content in the F-wave band $[9,10]$. Then, PSD estimates were obtained using four different methods: FFT, WLC, YWK and THM. Eventually, a new spectral index, the FWFR, was quantified to discriminate between HSs and AFPs. This approach is alternative to what found in literature [4-8], and was specifically designed not to rely on the P-wave identification and the rhythm irregularity. Indeed, automatic determination of the Pwave absence is still challenging (failure in detecting a $P$ wave doesn't necessarily imply P-wave absence), while the rhythm irregularity, although significantly different between HSs and AFPs (Table 1), is not specific for AF but may associate to other types of arrhythmia.

FWFR power in discriminating AFPs from HSs was high $\left(\mathrm{P}<10^{-11}\right.$; AUC $\left.\geq 85 \%\right)$ for all PSD methods. In order to provide a classification criterion possibly usable in clinics, OTHR was determined by minimizing an error function (Eq. 2) consisting in a weighted summation of FN and FP. Specifically, FP weight was set at half of FN one, since in clinical practice is more costly (in terms of clinical outcome) to detect an AFP as HS than vice versa. Accordingly, SE $(\geq 84 \%)$ was always higher than SP $(\geq 69 \%)$.

Although results obtained by using all four methods were all good and comparable (not statistically different from one another), we tend to prefer FFT-based approach not only for its simplicity, but also because high values of SE $(84 \%)$ and ACC (82\%) associate to the highest value of SP (78\%) and the narrowest CI (70-93\%). However, before a definitive identification of the best PSD method for AF identification by FWFR, some optimization procedures on the methods should be performed. Examples of these procedures include best window selection for FFT, application of zero-padding to increase PSD resolution, and others.

Overall, automatic AF identification by spectral analysis of F waves through FWFR evaluation represents an innovative and alternative tool to support diagnoses of atrial fibrillation. Its applicability to $10 \mathrm{~s}$ ECG combined with the neatness of FWFR computation are strengths that facilitate its introduction in the cardiologic clinical practice.

\section{Conclusion}

F-wave frequency evaluation by FWFR represents a promising clinical tool to automatically identify AF.

\section{References}

[1] S. S. Chugh et al., "Worldwide epidemiology of atrial fibrillation: A global burden of disease 2010 study," Circulation, vol. 129, no. 8, pp. 837-847, Feb. 2014.

[2] R. E. Klabunde, "Cardiovascular Physiology Concepts," Lippincott Williams \& Wilkins, p. 256, 2004.
[3] P. Kirchhof et al., "2016 ESC Guidelines for the management of atrial fibrillation developed in collaboration with EACTS," Europace, vol. 18, no. 11, pp. 1609-1678, Jan. 2016.

[4] J. Park, S. Lee, and M. Jeon, "Atrial fibrillation detection by heart rate variability in Poincare plot," Biomed. Eng. Online, vol. 8, pp.38, Dec. 2009.

[5] P. Pierleoni et al., "A eHealth System for Atrial Fibrillation Monitoring," Lect. Notes Electr. Eng., pp. 1-13, 2018. Ahead of print

[6] H. Pürerfellner et al., "P-wave evidence as a method for improving algorithm to detect atrial fibrillation in insertable cardiac monitors," Hear. Rhythm, vol. 11, no. 9, pp. 15751583, Sep. 2014.

[7] J. Blanc, "Automatic P-wave analysis of patients prone to atrial fibrillation," Med. Biol. Eng., vol. 40, no. 1, pp. 6371, Jan. 1998.

[8] A. Sbrollini et al., "AThrIA : a New Adaptive Threshold Identification Algorithm for Electrocardiographic P Waves Algorithm for Electrocardiographic P Waves," Comput. Cardiol., vol. 44, pp. 1-4, Sep. 2017.

[9] Q. Xi, A. V. Sahakian, J. Ng, and S. Swiryn, "Atrial fibrillatory wave characteristics on surface electrogram: ECG to ECG repeatability over twenty-four hours in clinically stable patients," J. Cardiovasc. Electrophysiol., vol. 15, no. 8, pp. 911-917, Aug. 2004.

[10] P. Sharma et al., "Surface ECG f Wave Analysis at Initial Onset of Paroxysmal and Persistent Atrial Fibrillation," $J$. Cardiovasc. Electrophysiol., vol. 28, no. 5, pp. 498-503, May 2017.

[11] A. P. Nemirko, and T. S. Lugovaya, "Biometric human identification based on electrocardiogram," in XII-th Russian Conference on Mathematical Methods of Pattern Recognition, pp. 387-390, Jun. 2005.

[12] A. L. Goldberger et al., "PhysioBank, PhysioToolkit, and PhysioNet: components of a new research resource for complex physiologic signals," Circulation, vol. 101, no. 23, pp. e215-e220, Jun. 2000.

[13] G. E. Moody, "Spontaneous termination of atrial fibrillation: a challenge from physionet and computers in cardiology 2004," Comput. Cardiol., pp. 101-104, Sep. 2004.

[14] J. Pan and W. J. Tompkins, "A Real-Time QRS Detection Algorithm," IEEE Trans. Biomed. Eng., vol. 32, no. 3, pp. 230-236, Mar. 1985.

[15] A. Agostinelli, C. Giuliani, and L. Burattini, "Extracting a Clean ECG from a Noisy Recording: a New Method based on Segmented-Beat Modulation," Comput. Cardiol., vol. 41, pp. 49-52, Sep. 2014.

[16] A. Agostinelli at al., "Segmented beat modulation method for electrocardiogram estimation from noisy recordings," Med. Eng. Phys., vol. 38, no. 6, pp. 560-568, Jun. 2016.

Address for correspondence.

Laura Burattini

Department of Information Engineering,

Università Politecnica delle Marche,

via Brecce Bianche 12, 60131, Ancona, Italy.

1.burattini@univpm.it 